\title{
Warm ischemic time' and renal function preservation in robotic partial nephrectomy - evaluating its real impact
}

\begin{abstract}
Objective: To evaluate the effect of warm ischemic time (WIT) on future renal function (RF) after robotic partial nephrectomy (RPN) and secondarily, also to find out predictors of future RF after RPN.

Method and materials: All patients who underwent RPN with normal pre-operative RF and normal contralateral kidney were included in the study except for those in whom oneyear follow up was not completed. Patients were divided in four groups based on WIT (zero, $<20$ minutes, 20 minutes to 30 minutes and $>30$ minutes). Comparison of demographic and perioperative parameters including follow-up up to one year were done. Univariable and multivariable analysis were done to detect significant correlation of RF with those parameters.
\end{abstract}

Results: Total 198 RPN patients were included in the study. The four groups had comparable demographic data (age, gender, comorbidity, smoking, body mass index, preoperative RF). Progressively increased WIT was significantly associated with increased tumour size $(p=0.022)$, RENAL nephrometry score $(p=0.003)$, operative time $(p=0.004)$ and blood loss $(p=0.046)$. Post-operatively, RF on first post-operative day $(p=0.627)$, at one month ( $p=0.581)$ and at one year $(p=0.378)$ had no significant difference between the four groups. Nine $(4.5 \%)$ patients progressed to chronic renal disease. Pre-operative RF and perioperative complications only had significant correlation with one-year RF.

Conclusion: Tumour size and RENAL nephrometry score significantly influenced WIT. Pre-operative RF and peri-operative complications were the only significant predictors of future RF and not the WIT.

Keywords: robotic partial nephrectomy, warm ischemic time, renal function, tumour size, RENAL nephrometry score
Volume 8 Issue 6 - 2020

\section{Krishnendu Biswas, Rohan S Batra,Abhishek \\ G Singh, Arvind P Ganpule, Ravindra B \\ Sabnis, Mahesh R Desai}

Urology Department, Muljibhai Patel Urological Hospital, India

Correspondence: Mahesh R Desai, MS, FRCS (Lon.), FRCS (Edn.), Senior Consultant and Managing Trustee, Muljibhai Patel Urological Hospital, DrVirendra Desai Road, Nadiad, Gujarat (India) - 38700I,India,Tel (+9I)982402804I,

Emailmrdesai@mpuh.org

Received: December 16, 2020 | Published: December 28, 2020
Abbreviations: RPN, robotic partial nephrectomy; RRP, residual renal parenchyma; NSS, nephron sparing surgery; RF, renal function; WIT, warm ischemic time; CIT, cold ischemic time; CECT, contrast enhanced computed tomography; eGFR, estimated glomerular filtration rate; $\mathrm{Hb}$, haemoglobin; $\mathrm{POD}$, post-operative day; OT, operative time; LOS, length of stay; BMI, body mass index; SICU, surgical intensive care unit; SFK, solitary functioning kidney; $\mathrm{CKD}$, chronic kidney disease; ESRD, end-stage renal disease

\section{Introduction}

Partial nephrectomy is now considered the treatment of choice for small renal masses (T1a), be it benign or malignant, and the spectrum has broadened to include larger masses as well, so long the operation is technically feasible with an acceptable residual renal parenchyma (RRP). ${ }^{1}$ As the technology advanced, robotic partial nephrectomy (RPN) became the preferred minimally invasive technique of choice for nephron sparing surgery (NSS). ${ }^{2}$ The idea of doing NSS is solely based on renal function (RF) preservation. ${ }^{3}$ Factors that determine RF following NSS may be non-modifiable (like tumour characteristics, patient profile) or modifiable (like surgical technique, RRP preserved). ${ }^{4}$ Needless to mention, modifiable factors are only under surgeon's control to improve the outcome. Warm ischemic time (WIT), as a modifiable factor, from prima facie, appears to hamper the post-operative RF. Theories like post-ischemic vasoconstriction with endothelial injury leading to inflammatory stigma, microvascular damage and reperfusion injury support the idea further. ${ }^{5}$ Hence, earlier researches were in the opinion to limit the WIT at least below 30 minutes ${ }^{6}$ or as low as possible. ${ }^{7}$ However, recent concept emphasizes on the quantity of the RRP after NSS. ${ }^{8,9}$ Few researches also inferred that the short-term fall in RF recovers in follow-up irrespective of WIT. ${ }^{10}$ Further, with the increasing popularity of robotic platform, more complex renal masses are subjected to NSS with possibilities of longer WIT. Hence, a robust evidence on the actual relationship between the WIT and the RF deterioration following RPN is essential. In our study, we primarily aimed to find out how much detrimental is the effect of WIT on future RF in patients with normal contralateral kidney after robotic NSS. Secondarily, we also aimed to find out the probable factors that may adversely affect the RF after robotic NSS

\section{Methods and materials}

\section{Study population}

All patients who underwent RPN at our centre since the start of robotic programme in September 2010 up to November 2019 were considered eligible for enlistment in the study, provided they have completed one year of follow-up after surgery. Patients who had solitary functioning kidney, who underwent excision of multiple tumours in the same kidney in the same sitting, patients with already compromised RF $\left(e G F R<60 \mathrm{ml} / \mathrm{min} / 1.73 \mathrm{~m}^{2}\right)$ and for whom imaging 
data in our records were not sufficient enough to calculate RENAL nephrometry score were excluded from the study. Patient who had post-operative re-exploration that required nephrectomy were also excluded from the study population. All surgeries were performed by three expert robotic surgeons at our institute with vast experience in robotic procedures. Institutional ethical committee clearance was taken before the study abiding the protocol of good clinical practices and ethical principles (Declaration of Helsinki). All original data are available with the authors for access.

\section{Surgical technique}

All patients underwent pre-operative contrast-enhanced computed tomography (CECT) of abdomen with renal angiography for tumour and vascular anatomy with 3D reconstruction (Figure 1). Routinely ipsilateral ureteric catheter was placed after anaesthesia. Surgical steps that were sequentially followed in all cases included ipsilateral colonic mobilization along with identification of ureter, gonadal vessels and psoas muscle; identification and mobilization of the renal artery and vein; mobilisation of the upper pole according to the need; demarcation of the tumour boundary with the help of intraoperative ultrasound; hilar clamping (renal artery and vein separately clamped); resection of the tumour; two layered renorrhaphy (Figure 2) with barbed suture with application of haemostatic agents (Gelatin granules with human thrombin and oxidised regenerated cellulose) and peri-renal drain placement. Zero-ischemia RPN were performed premeditatedly for cases with small $(<4 \mathrm{~cm})$ cortical renal mass (predominantly exophytic) in favourable positions (lateral, lower and upper pole) and where single isolated feeder vessel was well identified (Figure 3). Ipsilateral ureteric catheter was removed routinely on $2^{\text {nd }}$ post-operative day. We replaced the ureteric catheter with double-J stent where pelvicalyceal system were opened intraoperatively or tumour was larger one in vicinity to the pelvicalyceal system.
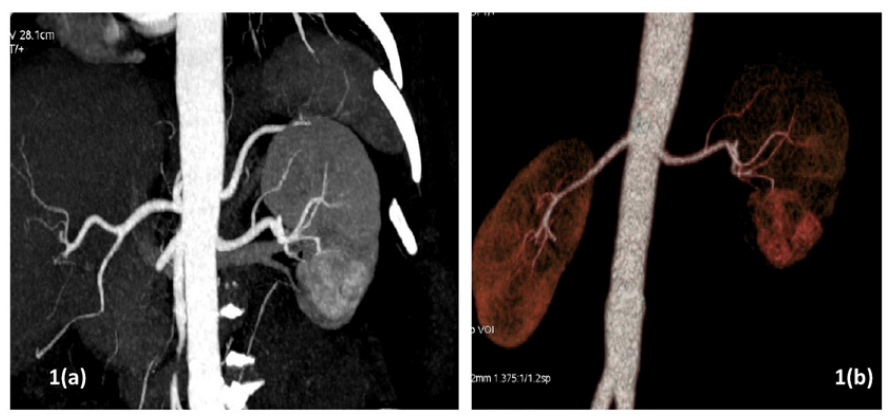

Figure I (a) Computed Tomography with renal angiography showing left lower pole renal mass; (b) 3D reconstruction.

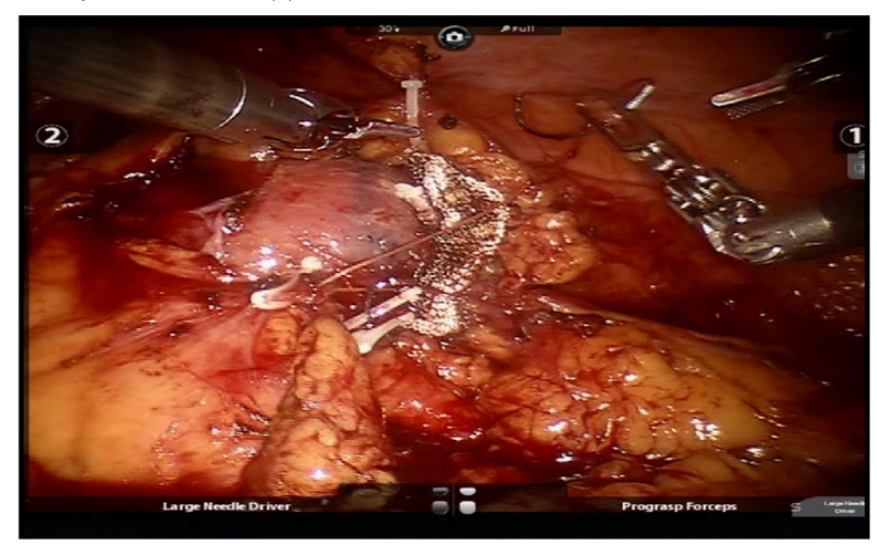

Figure 2 Renorrhaphy (barbed suture fixed with weck clips, oxidized regenerated cellulose mesh put at the raw site).
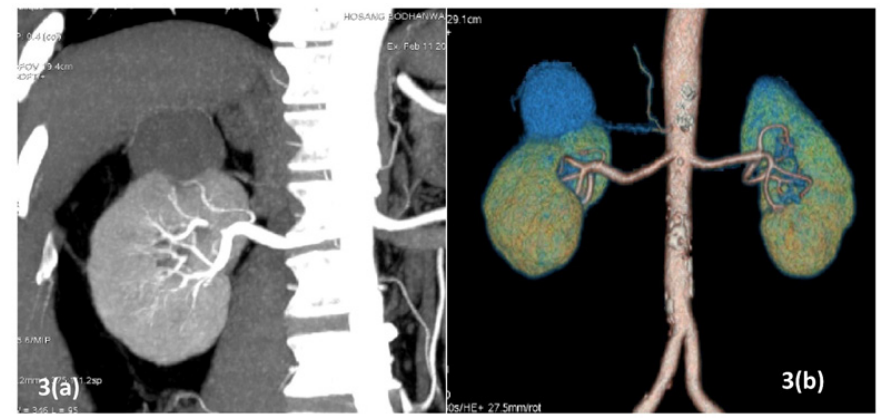

Figure 3 (a) right upper pole renal mass selected for zero ischemia (b) 3D reconstruction.

\section{Assessment}

Demographic data collected were age, gender, comorbidity (diabetes mellitus, hypertension), smoking habit and body mass index (BMI). Perioperative data included tumour characteristics (size, RENAL nephrometry score), pre-operative estimated glomerular filtration rate (eGFR) and haemoglobin $(\mathrm{Hb})$, total operative time (OT), WIT (time from application of hilar clamp to removal of hilar clamp), any intraoperative events, post-operative eGFR and $\mathrm{Hb}$ drop (estimated blood loss measured by the fall in $\mathrm{Hb}$ on POD1 after considering for blood transfusion, if transfused), any complication up to 30 days post-operatively following Clavein classification system and length of stay in hospital (LOS). Follow-up data included eGFR of first month and first year. Urine protein of first-year follow-up also tabulated. Clavein grade III or higher complications were considered major complications. The eGFR was calculated using the Modification of Diet in renal Disease (MDRD) formula. Patients were divided into four groups (based on WIT cut-off consensus in prior publications $\mathrm{s}^{7,11,12}$ ) namely 'zero-ischemia', 'WIT less than 20 minutes', 'WIT 20 minutes to 30 minutes' and 'WIT more than 30 minutes'. Comparison of the demographic and perioperative parameters were done to find out any significant difference between the groups. Analysis of the eGFR at one-month and at one-year (dependent variable) with various demographic and perioperative parameters (independent variables) were done to find out any correlation between them.

\section{Statistical analysis}

Categorical data and continuous data were compared with Chi square test and ANOVA (comparison of means) respectively. For factors predicting eGFR, univariable and multivariable linear regression analysis were used. Variables with $p<0.2$ on univariable analysis and those likely to be clinically significant were considered for multivariable analysis. Analysis was done in SPSS v.23 and $\mathrm{p}<0.05$ were considered significant.

\section{Results}

Total 256 RPN were performed at our institute from September 2010 to November 2019. Out of them, 32 patients were yet to reach one-year follow-up or did not completed one-year follow-up; 3 patients had RPN in solitary functioning kidney; 3 patients had multiple tumour excision in the concerned kidney in the same sitting; 14 patients had pre-existing RF impairment (eGFR $\left.<60 \mathrm{ml} / \mathrm{min} / 1.73 \mathrm{~m}^{2}\right) ; 5$ patients had inadequate imaging data and one patient had to undergo radical nephrectomy in re-exploration. Thus, a total of 58 patients were excluded and remaining 198 patients were included for assessment in the study. Table 1 depicted the demographic and perioperative data of all the patients. Twenty-two patients (11.1\%) had zero WIT, 64 
patients (32.3\%) had WIT less than 20 minutes, 84 patients (42.4\%) had WIT between 20 to 30 minutes and 28 patients (14.1\%) had WIT more than 30 minutes. The four groups had no statistically significant difference in terms of age, gender, BMI, co-morbidities, smoking habit and pre-operative eGFR. No patient had proteinuria pre-operatively. The mean tumour size in the 'zero-ischemic' group was $3.5 \pm 1.2 \mathrm{~cm}$ and only 2 patients (7\%) had RENAL nephrometry score more than 7. As the WIT increased the tumour size and RENAL nephrometry score significantly increased in each group. The post-operative $\mathrm{Hb}$ drop was highest for the zero-ischemic group $(1.9 \pm 1.2 \mathrm{gm} / \mathrm{dl})$. For the other three groups as the WIT increased, Hb drop also increased which was statistically significant. Baring the zero-ischemic group, all the three groups had progressively increased OT as the WIT increased. There was no statistically significant difference in the LOS in the four groups. In terms of post-operative complications, there was no statistically significant difference in the major complication (Clavein grade III or more) rate in the four groups. Overall, 17 patients $(8.5 \%)$ required one or more unit of blood transfusion.

Table I Demographic and Peri-operative Data

\begin{tabular}{|c|c|c|c|c|c|}
\hline & $\begin{array}{l}\text { Zero } \\
\text { ischemia }\end{array}$ & $\begin{array}{l}\text { WIT }<20 \\
\min \end{array}$ & $\begin{array}{l}\text { WIT } 20 \mathrm{~min} \text { to } 30 \\
\text { min }\end{array}$ & $\begin{array}{l}\text { WIT >30 } \\
\min \end{array}$ & $\mathbf{p}$ \\
\hline No of Patients (\%) & $22(11.1)$ & $64(32.3)$ & $84(42.4)$ & $28(14.1)$ & \\
\hline Age (years); mean \pm SD & $53.3 \pm 10.3$ & $52.4 \pm 11.4$ & $50.3 \pm 14.1$ & $48.8 \pm 12.5$ & 0.251 \\
\hline Male Gender (\%) & $16(72.7)$ & $40(62.5)$ & $68(80.9)$ & $19(67.8)$ & 0.091 \\
\hline BMI; mean \pm SD & $26.3 \pm 2.7$ & $26.1 \pm 2.9$ & $25.8 \pm 3.4$ & $25.8 \pm 3.9$ & 0.894 \\
\hline Comorbidity (with DM or HTN or both) (\%) & $10(45.4)$ & $23(35.9)$ & $33(39.2)$ & $10(35.7)$ & 0.864 \\
\hline Smoking (\%) & $4(8.8)$ & $16(25)$ & $17(20.2)$ & $2(5.6)$ & 0.264 \\
\hline Tumour Size $(\mathrm{cm}) ;$ mean \pm SD & $3.5 \pm 1.2$ & $3.9 \pm 1.7$ & $4.4 \pm 1.5$ & $4.7 \pm 1.7$ & 0.022 \\
\hline RENAL Nephrometry Score > 7 (\%) & $2(9.0)$ & $15(23.4)$ & $23(27.3)$ & $12(42.8)$ & 0.003 \\
\hline Pre-operative eGFR $(\mathrm{mi} / \mathrm{min} / \mathrm{l} .73 \mathrm{~m} 2)$; mean \pm SD & $100.3 \pm 28.4$ & $106.5 \pm 25.0$ & $100.6 \pm 24.1$ & $107.2 \pm 25.3$ & 0.419 \\
\hline Operative Time (min); mean \pm SD & $182.7 \pm 55.6$ & $150.7 \pm 43.0$ & $|72| \pm 53.9$. & $188.2 \pm 59.5$ & 0.004 \\
\hline WIT (min); mean \pm SD & 0 & $15.2 \pm 3.1$ & $23.4 \pm 3.3$ & $35.1 \pm 4.6$ & $<0.001$ \\
\hline Post-operative Hb drop (gm/dl); mean \pm SD & $1.9 \pm 1.2$ & $1.3 \pm 1.0$ & $1.3 \pm 0.9$ & $1.6 \pm 1.0$ & 0.046 \\
\hline Length of Stay (day); mean \pm SD & $5.4 \pm 1.1$ & $5.5 \pm 1.0$ & $6.1 \pm 1.7$ & $5.8 \pm 1.3$ & 0.091 \\
\hline Major complications (Clavein Grade $\geq \mathrm{III}$ ) (\%) & $\mathrm{I}(2.2)$ & $5(7.8)$ & $8(9.5)$ & $2(5.6)$ & 0.886 \\
\hline eGFR on PODI (ml/min/l.73m2); mean \pm SD & $78.7 \pm 25.2$ & $84.6 \pm 23.1$ & $80.6 \pm 21.3$ & $83.2 \pm 21.0$ & 0.627 \\
\hline eGFR at I Month $(\mathrm{ml} / \mathrm{min} / 1.73 \mathrm{~m} 2)$; mean \pm SD & $91.3 \pm 24.1$ & $95.6 \pm 22.5$ & $93.0 \pm 20.7$ & $98.4 \pm 21.3$ & 0.581 \\
\hline eGFR at I Year D $(\mathrm{ml} / \mathrm{min} / 1.73 \mathrm{~m} 2)$; mean $\pm S D$ & $88.1 \pm 23.3$ & $94.7 \pm 23.0$ & $88.9 \pm 20.6$ & $91.8 \pm 18.8$ & 0.378 \\
\hline CKD III or more at I year (eGFR < 60ml/min/I.73m2) (\%) & $2(4.4)$ & $3(4.6)$ & $3(3.5)$ & $\mathrm{I}(3.5)$ & 0.73 \\
\hline Proteinuria at I Year (\%) & $4(18.1)$ & $7(10.9)$ & $8(9.5)$ & $3(10.7)$ & 0.721 \\
\hline
\end{tabular}

Among major complications 6 patients had urine leak, 3 patients had perirenal hematoma, 3 patients had paralytic ileus, 2 patients had sepsis, 1 patient had pulmonary embolism, 1 patient had pleural effusion. In all six patients with urine leak 'double J' stent was placed and kept for 6 weeks and they recovered well. Three patients with hematoma underwent re-exploration and only in one patient significant bleeder was found. All the three re-explored patients recovered satisfactorily. Rest of the 7 patients with major complications recovered gradually after meticulously conservative management in surgical intensive care unit (SICU). There was no death in our study population. Overall, patients suffered initial fall in eGFR on first postoperative day (average 19.2\% fall) followed by rise and stabilization of eGFR at one-month (average $7.0 \%$ fall from pre-operative eGFR) and one-year follow-up (average $10.4 \%$ fall from pre-operative eGFR) Table 1 but these fall in eGFR were not significantly different in the four groups. In Table 2 comparison of absolute fall in eGFR at one-year (from pre-operative eGFR) between various 'WIT groups' clearly showed no statistically significant difference of eGFR change between four WIT groups. In our study, after one year, total 9 patients (4.5\%) progressed to stage III or more CKD and 22 patients $(11.1 \%)$ showed presence of proteinuria (1+ or more) but no specific group showed any higher propensity to develop CKD or proteinuria. At one-month follow-up (Table 3) \& (Figure 4) on univariable analysis age, co-morbidity and pre-operative eGFR had statistically significant correlation with one-month-eGFR. However, in multivariable model, only pre-operative eGFR significantly correlated with the one-montheGFR. Similarly, at one year of follow up (Table 3) \& (Figure 4) on univariable analysis along with age, co-morbidity and pre-operative eGFR also pre-operative BMI and major complications (grade III or more in Clavein grade) were the variables that significantly correlated with one-year-eGFR. On multivariable model, only pre-operative eGFR and major complications significantly correlated with the oneyear-eGFR. 
Table 2 Comparison of Change in eGFR at one-year follow-up between various 'WIT groups'

\begin{tabular}{|c|c|c|}
\hline WIT groups & Fall in eGFR at one-year $(\mathrm{ml} / \mathrm{min} / 1.73 \mathrm{~m} 2)$; mean \pm SD & $\mathbf{p}$ \\
\hline Zero ischemia vs WIT $<20 \mathrm{~min}$ & $12.1 \pm 15.3$ vs $11.7 \pm 16.6$ & 0.927 \\
\hline Zero ischemia vs WIT $20 \mathrm{~min}$ to $30 \mathrm{~min}$ & $12.1 \pm 15.3$ vs $11.8 \pm 16.7$ & 0.952 \\
\hline Zero ischemia vs WIT > $30 \mathrm{~min}$ & $12.1 \pm 15.3$ vs $15.4 \pm 18.1$ & 0.495 \\
\hline WIT $<20 \mathrm{~min}$ vs WIT $20 \mathrm{~min}$ to $30 \mathrm{~min}$ & $11.7 \pm 16.6$ vs $11.8 \pm 16.7$ & 0.962 \\
\hline $\mathrm{WIT}<20 \mathrm{~min}$ vs WIT $>30 \mathrm{~min}$ & $11.7 \pm 16.6$ vs $15.43 \pm 18.1$ & 0.343 \\
\hline WIT $20 \mathrm{~min}$ to $30 \mathrm{~min}$ vs WIT $>30 \mathrm{~min}$ & $1 \mathrm{I} .8 \pm 16.7$ vs $15.43 \pm 18.1$ & 0.342 \\
\hline
\end{tabular}

Table 3 Correlation of eGFR at one-month and at one-year with various parameters

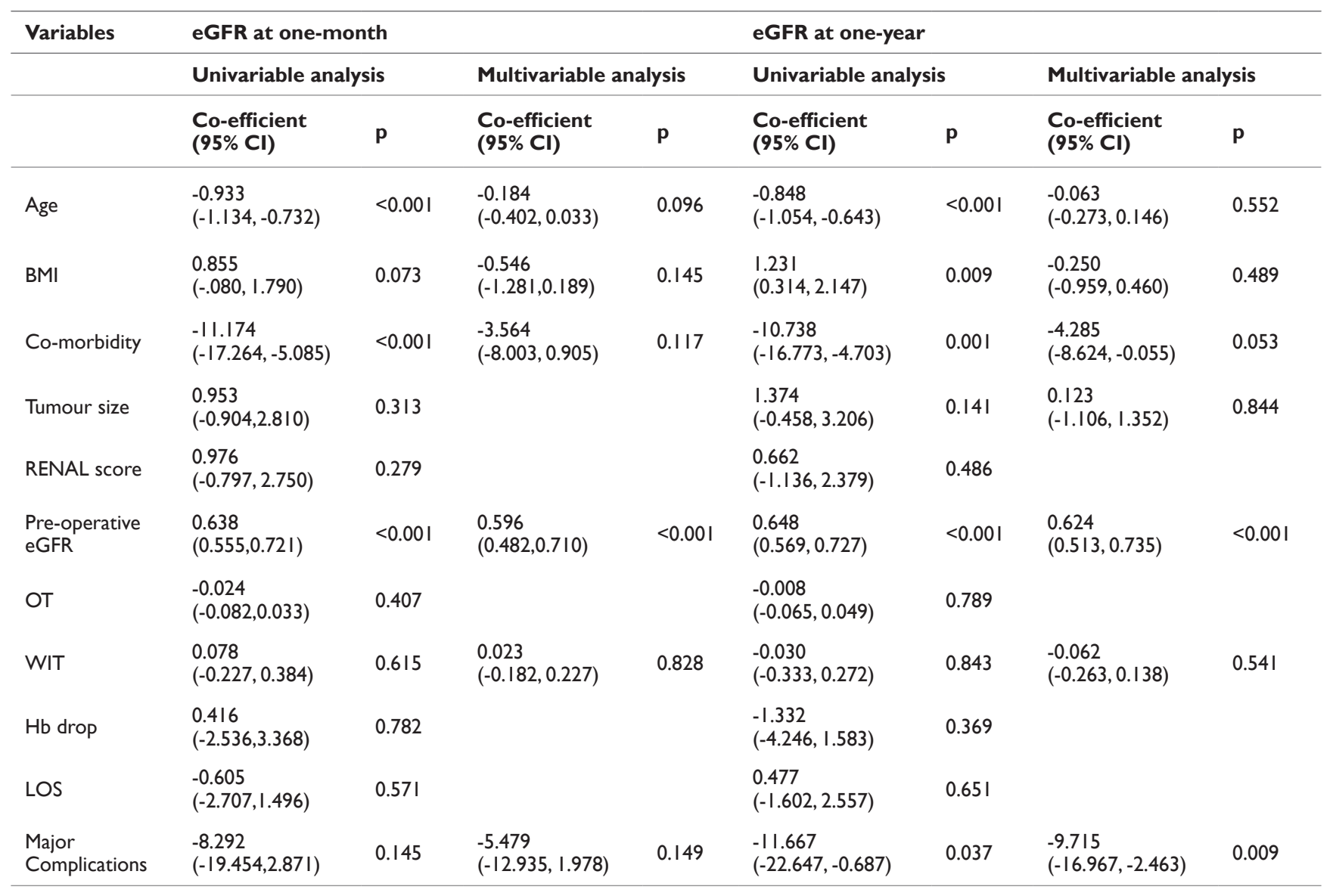
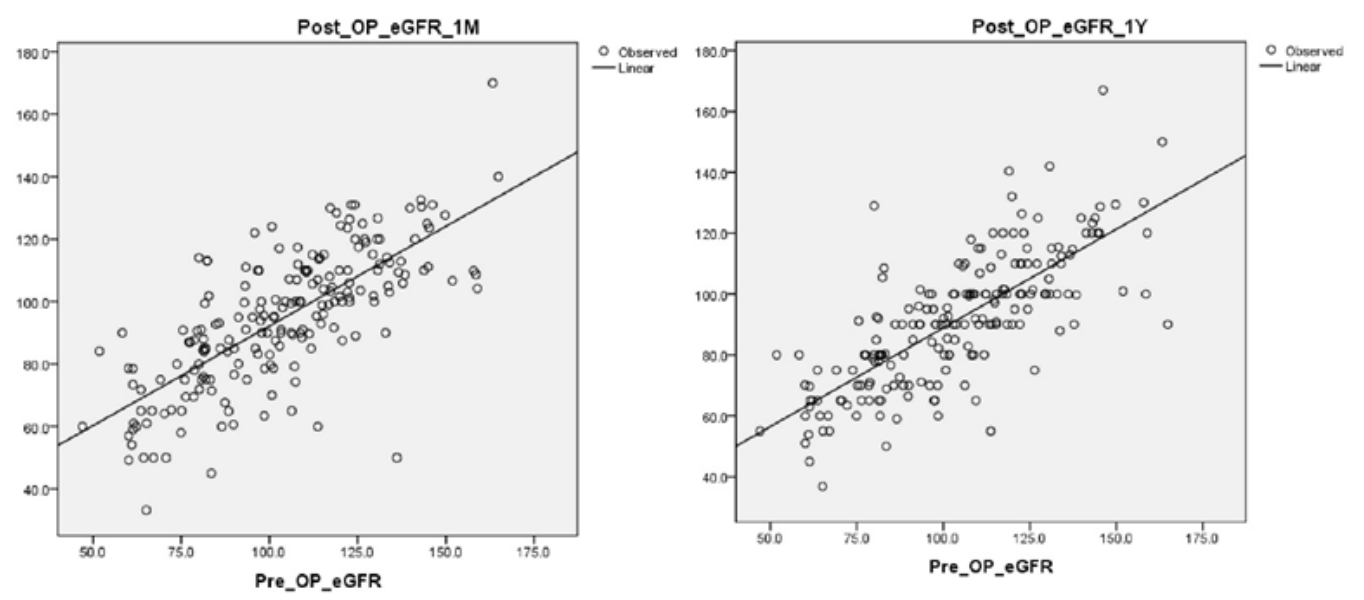

Figure 4 Scatter plot of post-operative eGFR at (a) one-month and at (b) one-year vs pre-operative eGFR.

Citation: Biswas K, Batra RS, Singh AG, et al.Warm ischemic time' and renal function preservation in robotic partial nephrectomy - evaluating its real impact. Urol Nephrol Open Access J. 2020;8(6): I6I-166. DOI: 10.15406/unoaj.2020.08.00298 


\section{Discussion}

The concept of NSS for management of renal tumour evolved with the goal of preservation of RF without violating the principles of oncology. ${ }^{13}$ Data from the literature suggest, an overall incidence of end-stage renal disease of $2.5 \%$ with subcategorised incidence of $0.1 \%, 3.7 \%$ and $36 \%$ respectively for patients with normal RF, stage III CKD and stage IV CKD after NSS. ${ }^{4}$ Researcher have divided the possible adverse factors into 'modifiable' and 'non-modifiable' groups. ${ }^{4,14}$ The non-modifiable factors (demographic features, tumour characteristic) are beyond the control of the surgeon, but modifiable factor in the form of WIT has long been a matter of research topic among urologists. Clamping the renal hilum eases dissection making the operative field bloodless but may prompt acute tubular necrosis, microvascular damage, reperfusion injury to the kidney. ${ }^{5}$ Although Parekh et al. ${ }^{15}$ have pointed out remarkable resistance of the kidney against longer WIT, but most articles in the literature favoured a reasonably shorter WIT. Thompson et al. ${ }^{7,11}$ recommended a preferred WIT of less than 20 minutes, with 25 minutes as a critical cut-off. The dictum 'every minute counts' held ground well until the concept of parenchyma preservation gained popularity. The RF is the summation of the functions of all the nephrons taken together, hence greater the RRP after NSS, greater will be the chance of preserved RF. Volpe et al. ${ }^{16}$ emphasised the importance of RRP in maintaining RF and also discussed importance tumour resection technique and renorrhaphy which were deemed equally important for optimum RRP preservation. One study had shown, a 5\% increase in renal volume preservation minimizes the risk of de-novo stage IV CKD by $17 \% .{ }^{17}$

In our retrospective study of 198 patients, we excluded patients with compromised RF (eGFR $<60 \mathrm{ml} / \mathrm{min} / \mathrm{m}^{2}$ ) as we wanted to find out the outcome in patients having normal pre-operative RF with contralateral normal kidney. Pre-existing CKD as a predictor of further RF deterioration is well known in literature. ${ }^{4,17}$ Tumour size and the RENAL nephrometry score were the indirect indicators of operative difficulty. Both the WIT and the OT increased as those parameters increased. Kwang Jin Ko et al..$^{18}$ concluded that tumour size, PADUA score and surgeon's experience were important determining factors for longer WIT. We did not calculate PADUA score, instead following Altunrende et al. ${ }^{19}$ we calculated RENAL nephrometry score and found similar results. Highest blood loss in the zero-ischemic group was understandably due to the off-clamp nature of the surgery and for the rest of the three groups as the WIT increased, Hb drop also increased. This has been well explained by Zargar et al. ${ }^{20}$ as more complex masses (higher RENAL nephrometry score) required more complex dissection and renorrhaphy, hence increased WIT and blood loss. The significant increase in OT with increased WIT and tumour complexity can also be explained by the same logic. We did not find any statistically significant difference of complications among various groups. The lowest incidence of complications in the zero-ischemic group, however, may be a biased outcome because of selection of smaller and less complex renal mass.

The causes of overall fall in eGFR (19.2\%) on postoperative dayone may be prerenal (e.g. hypotension, volume depletion), intrinsic renal (e.g. acute tubular necrosis, nephron mass loss) or even post renal (e.g. clot obstruction). We routinely do CECT abdomen with renal angiography one day prior to surgery, hence, transient contrastinduced fall of eGFR cannot be ruled out. The eGFR in follow-up at one-month (fall by $7.0 \%$ ) and one-year (fall by $10.4 \%$ ) showed recovery and stabilisation. Overall, rate of progression to CKD III or more was $4.5 \%$ at one year with development of new proteinuria in $11.1 \%$ patients both of which are comparable with literature. ${ }^{4}$ Wang et al. ${ }^{21}$ in a similar study found a mean decrease in eGFR of $20.3 \%$ and $11.3 \%$ at first-day and at first-year after surgery with trend towards stable eGFR from third-month onwards. Similar results were also noted by Lane et al. ${ }^{4}$ and Porpiglia et al. ${ }^{22}$ In our study (Table 2), the change in eGFR was not statistically significant between various WIT groups and correlation of one-month and one-year eGFR with various parameters (Table 3) conclusively proved pre-operative eGFR as the major determining factor with statistical significance. Interestingly, peri-operative major complications turned out to be an additional correlating factor of one-year RF. Although there are no supporting data in existing literature for this outcome; urine leak, major hematoma or sepsis may induce permanent and long-term renal damage from theoretical point of view.

Literature on the impact of WIT in robotic NSS are relative sparse compared to open or laparoscopic NSS, although the concept remains the same. Thompson et al. ${ }^{7,11,17}$ gave much emphasis on WIT as a predictor of short term and long-term RF, particularly in open NSS in SFK setting and also included pre-operative eGFR and RRP as independent predictor of new-onset stage IV CKD. Lane et al., ${ }^{4,2,23}$ in large multi-institutional studies on SFK described age, tumour size, pre-operative eGFR and WIT as predictors of RF with preoperative eGFR being the most important parameter. Simmons et al. ${ }^{8}$ also considered preoperative RF and RRP as the sole determinants for long term RF. Parekh et al. ${ }^{15}$ proved that acute kidney injury biomarkers and RF did not correlate with ischemia time. Shikanov et al. ${ }^{24}$ reiterated that long term global RF was not impacted by WIT in NSS. In a propensity matched comparative study by Lee et al. ${ }^{25}$ the conclusion also favoured our outcome strongly that WIT was not the significant determinant of long-term RF. On the contrary, works of Funahashi et al. ${ }^{26}$, Rosen et al. ${ }^{27}$ strongly supported WIT as the most important predictors of RF. Wang et al. ${ }^{21}$ in their article inferred that both pre-operative RF and WIT were significant determinant of RF. Interestingly, Chen et al. ${ }^{28}$ and Song et al. ${ }^{29}$ in their articles mentioned age, tumour size, tumour location, RRP and procedure type as predictors of long-term RF and neither pre-operative eGFR nor WIT was a statistically significant predictor.

In our study, the long-term RF in the 'zero-ischemic' group were also similar to the other WIT groups. Smith et al. ${ }^{30}$ showed similar RF outcome between off-clamp and on-clamp NSS. In patients with contralateral normal kidney, compensatory hypertrophy of the contralateral kidney may reveal overall satisfactory function masking the decline in the function of the ipsilateral kidney. Pouliot et al. ${ }^{31}$ using renal scans to precisely measure the RF had shown a WIT cutoff more than $25 \mathrm{~min}$ were detrimental for the suffered kidney. We have not done any isotope renal scan and used the MDRD formula to estimate the global RF. Preservation of renal parenchyma as much as possible is an important predictor of future RF. ${ }^{8}$ The volume excised can be assessed by manual estimation or by imaging studies for better prediction..$^{9,28,29}$ Recently, Haifler et al. ${ }^{32}$ showed that renal tumour contact area with the kidney surface was an independent predictor of RF after NSS. We did not do any excised volume estimation or contact surface area estimation in our study. The entire details of WIT and RF preservation have been meticulously illustrated by Volpe et al. ${ }^{16}$ in a collaborative review where final emphasis was given upon pre-operative RF and RRP that govern the long-term RF. WIT and RRP both reciprocate each other and both are modifiable factor under surgeon's control. ${ }^{14}$ But, pre-operative RF (eGFR) remains unquestionably the most important non-modifiable factors for RF prediction. The probable limitations of our study may be the retrospective nature of the study. Our study population included only 198 patients from a single institute. We have not measured the RRP 
preserved which in contemporary literature appears to be the most important determining factor for RF estimation. In bilateral kidney setup, using MDRD formula we have estimated the global RF; use of renal scan could have better estimated the RF of the concerned kidney. Many new techniques are described in literature e.g., early declamping, artery only clamping, selective or super selective clamping. Our data does not include such specification.

\section{Conclusion}

The size of the renal tumour and the RENAL nephrometry score were significantly associated the WIT in RPN. WIT was not the predictor of RF at one-month and one-year of follow-up. Most important determinant of RF was the 'pre-operative eGFR'. Perioperative complication had significant correlation with one-year RF.

\section{Acknowledgments}

None.

\section{Conflicts of interest}

The author declares there is no conflict of interest.

\section{References}

1. Simmons MN, Chung BI, Gill IS. Perioperative efficacy of laparoscopic partial nephrectomy for tumours larger than $4 \mathrm{~cm}$. Eur Urol. 2009a;55(1):199-208.

2. Khalifeh A, Autorino R, Eyraud R, et al. Three-year oncologic and renal functional outcomes after robot-assisted partial nephrectomy. Eur Urol. 2013;64(5):744-750.

3. Campbell SC, Novick AC, Belldegrun A, et al. Guideline for management of the clinical T1 renal mass. J Urol. 2009;182(4):1271-1279.

4. Lane BR, Babineau DC, Poggio ED, et al. Factors predicting renal functional outcome after partial nephrectomy. $J$ Urol. 2008;180(6):23632368 .

5. Derweesh IH, Novick AC. Mechanisms of renal ischaemic injury and their clinical impact. BJU Int. 2005;95(7):948-950.

6. Novick AC. Renal hypothermia: in vivo and ex vivo. Urol Clin North Am 1983;10: 637-644.

7. Thompson RH, Lane BR, Lohse CM, et al. Every minute counts when the renal hilum is clamped during partial nephrectomy. Eur Urol. 2010;58:340-345.

8. Simmons MN, Fergany AF, Campbell SC. Effect of parenchymal volume preservation on kidney function after partial nephrectomy. $J$ Urol. 2011;186(2):405-410.

9. Mir MC, Campbell RA, Sharma N, et al. Parenchymal volume preservation and ischemia during partial nephrectomy: functional and volumetric analysis. Urology. 2013;82(2):263-268.

10. Selcuk Erdem, Abubekir Boyuk, Tzevat Tefik, et al. Warm IschemiaRelated Postoperative Renal Dysfunction in Elective Laparoscopic Partial Nephrectomy Recovers During Intermediate-Term Follow-Up. $J$ Endourol. 2015 Sep;29(9):1083-1090.

11. Thompson RH, Frank I, Lohse CM, et al. The impact of ischemia time during open nephron sparing surgery on solitary kidneys: a multiinstitutional study. J Urol. 2007;177(2):471-476.

12. Lane BR, Fergany AF, Weight CJ, Campbell SC. Renal functional outcomes after partial nephrectomy with extended ischemic intervals are better than after radical nephrectomy. J Urol. 2010;184(4):1286-1290.

13. Scosyrev E, Messing EM, Sylvester R, et al. Renal function after nephronsparing surgery versus radical nephrectomy: results from EORTC randomized trial 30904. Eur Urol. 2014;65(2):372-377.
14. James Porter. Renal Ischemia During Partial Nephrectomy: Does Every Minute Still Count? Eur Urol. 2015;68(1):75-76.

15. Parekh DJ, Weinberg JM, Ercole B, et al. Tolerance of the human kidney to isolated controlled ischemia. J Am Soc Nephrol. 2013;24: 506-517.

16. Alessandro Volpe, Michael L Blute, Vincenzo Ficarra, et al. Renal Ischemia and Function After Partial Nephrectomy: A Collaborative Review of the Literature. European Urology. 2015;68(1):61-74.

17. Thompson RH, Lane BR, Lohse CM, et al. Renal function after partial nephrectomy: effect of warm ischemia relative to quantity and quality of preserved kidney. Urology. 2012;79(2):356-360.

18. Kwang Jin Ko, Don Kyoung Choi, Seung Jea Shin, et al. Predictive factors of prolonged warm ischemic time ( $\geq 30$ minutes) during partial nephrectomy under pneumoperitoneum. Korean J Urol. 2015;56(11):742748.

19. Altunrende F, Laydner H, Hernandez AV, et al. Correlation of the RENAL nephrometry score with warm ischemia time after robotic partial nephrectomy. World J Urol. 2013;31(5):1165-1169.

20. Homayoun Zargar, Oktay Akca, Daniel Ramirez, et al. The impact of extended warm ischemia time on late renal function after robotic partial nephrectomy. J Endourol. 2015;29(4):444-448.

21. Zhixian Wang, Chang Liu, Ruibao Chen, et al. Will the kidney function be reduced in patients with renal cell carcinoma following laparoscopic partial nephrectomy? Baseline eGFR, warm ischemia time, and RENAL nephrometry score could tell. Urol Oncol. 2018;36(11):498.e15-498.e24.

22. Porpiglia F, Fiori C, Bertolo R, et al. Long-term functional evaluation of the treated kidney in a prospective series of patients who underwent laparoscopic partial nephrectomy for small renal tumors. Eur Urol. 2012;62:130-135.

23. Lane BR, Russo P, Uzzo RG, et al. Comparison of cold and warm ischemia during partial nephrectomy in 660 solitary kidneys reveals predominant role of nonmodifiable factors in determining ultimate renal function. $J$ Urol. 2011;185:421-427.

24. Shikanov S, Lifshitz D, Chan AA, et al. Impact of ischemia on renal function after laparoscopic partial nephrectomy: a multicenter study. $J$ Urol. 2010;183: 1714-1718.

25. Hakmin Lee, Byung D Song, Seok-Soo Byun, et al. Impact of warm ischaemia time on postoperative renal function after partial nephrectomy for clinical T1 renal cell carcinoma: a propensity score-matched study. BJU Int. 2018;121(1):46-52.

26. Funahashi $Y$, Hattori R, Yamamoto $T$, et al. Ischemic renal damage after nephron-sparing surgery in patients with normal contralateral kidney. Eur Urol. 2009;55:209-215.

27. Daniel C Rosen, Muthumeena Kannappan, David J Paulucci, et al. Reevaluating Warm Ischemia Time as a Predictor of Renal Function Outcomes After Robotic Partial Nephrectomy. Urology. 2018;120:156161.

28. Chan AA, Wood CG, Caicedo J, et al. Predictors of unilateral renal function after open and laparoscopic partial nephrectomy. Urology. 2010;75:295-302.

29. Song C, Bang JK, Park HK, Ahn H. Factors influencing renal function reduction after partial nephrectomy. J Urol. 2009;181:48-53.

30. Smith GL, Kenney PA, Lee Y, et al. Non-clamped partial nephrectomy: techniques and surgical outcomes. BJU Int. 2011;107:1054-1058.

31. Pouliot F, Pantuck A, Imbeault A, et al. Multivariate analysis of the factors involved in loss of renal differential function after laparoscopic partial nephrectomy: a role for warm ischemia time. Can Urol Assoc $J$. 2011;5:89-95.

32. Miki Haifler, Benjamin T Ristau, et al. External Validation of Contact Surface Area as a Predictor of Postoperative Renal Function in Patients Undergoing Partial Nephrectomy. J Urol. 2018;199(3):649-654. 